\title{
Previous cultures are not clinically useful for guiding empiric antibiotics in suspected ventilator-associated pneumonia: Secondary analysis from a randomized trial ${ }^{i s}$
}

\author{
Kevin M. Sanders ${ }^{a}$, Neill K.J. Adhikari ${ }^{a}$, Jan 0. Friedrich ${ }^{a}$, Andrew Day ${ }^{b}$, \\ Xuran Jiang ${ }^{\mathbf{b}}$, Daren Heyland ${ }^{\mathrm{b}, *}$, \\ for the Canadian Critical Care Trials Group
}

\author{
${ }^{a}$ Interdepartmental Division of Critical Care Medicine, University of Toronto, Toronto, Ontario, Canada M5T 2S8 \\ ${ }^{\mathrm{b}}$ Department of Medicine, Clinical Evaluation Research Unit, Kingston General Hospital, Queens University, \\ Kingston, Ontario, Canada K7L 2 V7
}

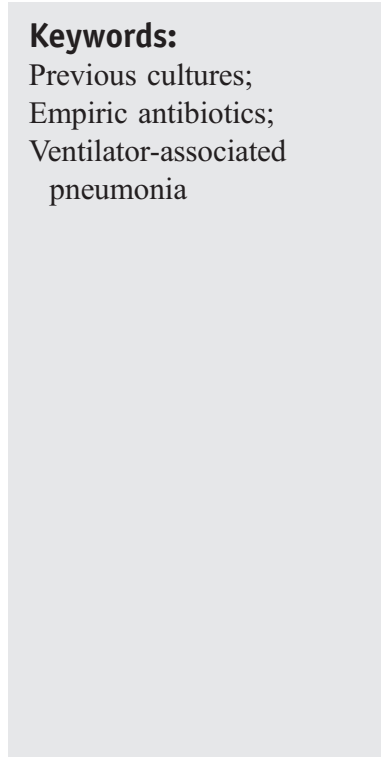

\begin{abstract}
Purpose: To examine the predictive validity of prior cultures at predicting the microorganism isolated at the time of suspicion of ventilator-associated pneumonia (VAP).

Materials and Methods: We performed a retrospective analysis of a randomized controlled trial of different diagnostic and antibiotic strategies. In a subset of patients with pre-enrollment cultures, we examined agreement between cultures 1 to 3 days before suspicion of VAP and enrollment cultures performed on the day of suspicion of VAP and potential antibiotic error rates (estimated using the equation 1 - crude agreement).

Results: Two hundred eighty-one (39\%) of 739 patients had pre-enrollment culture. One hundred thirty $(46 \%)$ of 281 yielded a pathogenic microorganism. In patients with positive pre-enrollment cultures, crude agreement was 0.63 (95\% confidence interval, 0.55-0.71) for organism, $0.84(0.77-0.89)$ for Gram class, and $0.61(0.52-0.69)$ for species with sensitivity. Potential antibiotic error rates ranged from $16 \%$ $(11 \%-33 \%)$ to $39 \%(31 \%-48 \%)$. Better agreement $(P=.033)$ occurred in isolates from patients receiving new antibiotics during the surveillance period (0.78 [0.64-0.87]) compared to those not on antibiotics (0.58 [0.45-0.69]), or on no new antibiotics (0.50 [0.32-0.68]).

Conclusions: There is poor agreement between prior cultures and cultures performed at time of suspicion of VAP. Prior cultures should not be used to narrow the spectrum of empiric antibiotics. (C) 2008 Elsevier Inc. All rights reserved.
\end{abstract}

Supported by grants from the Canadian Institutes of Health Research and Physicians' Services, Inc, of Ontario, and by unrestricted grants from AstraZeneca and Bayer.

* Corresponding author. Tel.: +1 613549 6666x3339; fax: +1 613548 1351.

E-mail address: dkh2@queensu.ca (D. Heyland).

\section{Introduction}

Ventilator-associated pneumonia (VAP) is a common nosocomial infection in mechanically ventilated patients, with an estimated incidence of $8 \%$ to $28 \%$ [1]. Ventilator- 
associated pneumonia is associated with an increased duration of mechanical ventilation, length of hospital stay, and mortality [2-4]. Several observational studies suggest that the outcome of VAP is affected by the adequacy of initial antibiotics [5,6]. The American Thoracic Society guidelines recommend initial treatment with broad-spectrum antibiotics and subsequent rapid de-escalation to ensure adequacy of antimicrobial coverage [7]. However, there are growing concerns that the overuse of broad-spectrum antibiotics in intensive care units (ICUs) increases bacterial resistance, length of stay, and mortality $[8,9]$.

An alternative approach to prescribing initial broadspectrum antibiotics for VAP is to regularly collect respiratory specimens in mechanically ventilated patients, regardless of clinical status, to document the presence of potentially pathogenic microorganisms. Basing empiric antibiotic choices on the results of these surveillance cultures has the potential advantage of prescribing narrower spectrum antibiotics, provided that surveillance cultures accurately reflect pathogens isolated at time of suspected VAP. Although several studies support the predictive accuracy of surveillance cultures [10-12], others do not [13,14].

We performed a retrospective analysis of a recent multicenter randomized controlled trial (RCT) of diagnostic strategies and antibiotic selection in VAP $[15,16]$ to assess the reliability of previous cultures to guide empiric antibiotic therapy. Our main objective was to answer the following clinically important question: could empiric antimicrobial therapy guided by the results of previous respiratory tract cultures adequately treat organisms grown from cultures performed at the time of a suspicion of VAP?

\section{Methods}

We performed a retrospective analysis of data from a RCT, details of which have been published previously [15]. The study enrolled 740 patients from 28 ICUs in Canada and the United States between May 2000 and February 2005. The trial used a $2 \times 2$ factorial design to assess the impact on mortality of bronchoalveolar lavage (BAL) vs endotracheal aspirates (ETA) [15] and broad-spectrum combination antibiotic therapy (meropenem and ciprofloxacin) vs monotherapy (meropenem) [16] on mortality among mechanically ventilated patients with suspected VAP.

\subsection{Participants}

Patients were eligible for the study if they had received mechanical ventilation for at least 4 days and if they had suspected VAP. Pneumonia was defined by new or persistent radiographic features of pneumonia without another obvious cause and any 2 of the following clinical features: a temperature exceeding $38^{\circ} \mathrm{C}$, leukocytosis (defined as a leukocyte count exceeding $11.0 \times 10^{3}$ per cubic millimeter) or neutropenia (defined as a neutrophil count of $<3500$ per cubic millimeter), purulent endotracheal secretions, potentially pathogenic bacteria isolated from the ETA, and increasing oxygen requirements. Patients were excluded if they were immunocompromised; unsuitable for bronchoscopy; allergic to penicillins, cephalosporins, carbapenems, or ciprofloxacin; infected or colonized with pseudomonas species or methicillin-resistant Staphylococcus aureus; recipients of study drugs (ciprofloxacin within 24 hours and meropenem within 7 days before enrollment); expected to die or undergo withdrawal of treatment within 72 hours after enrollment; unlikely to leave the ICU within 3 weeks; pregnant or lactating; or previously enrolled in this or another interventional trial. Written informed consent was obtained from the substitute decision maker of all enrolled patients.

\subsection{Microbiological specimens}

Microbiological specimens were obtained from all patients at the time of enrollment. Specimens consisted of either a quantitative culture of BAL fluid or a nonquantitative culture of an ETA, depending on patient assignment. Routine surveillance cultures were not mandated, but previous nonquantitative ETAs were performed at the discretion of the physician. Any ETA taken 1 to 3 days before enrollment qualified as a previous culture, whereas an ETA taken on the same day as enrollment was pooled with the enrollment culture. Bronchoalveolar lavage specimens taken 1 to 3 days before enrollment were excluded from all analyses because there were very few $(n=18)$. A positive culture was defined as any growth of a pathogenic organism on BAL or ETA regardless of colony count. Specimens yielding Candida, coagulase-negative Staphylococci, or normal flora were considered nonpathogenic and were classified as no growth requiring antibiotic treatment for both the previous and enrollment cultures. After discharge or death, site investigators reviewed hospital records, incorporating culture results, response to antibiotics, and other features of the clinical course to adjudicate whether study patients had developed VAP and to determine the final clinical and microbiological outcomes using standard definitions [15].

\subsection{Outcomes}

The primary outcome for the present analysis was crude agreement in patients with a positive previous culture. This was defined by isolation of no new organisms from enrollment cultures compared to what was found in previous cultures. The definition of the primary outcome was selected to reflect the ability of prior cultures to guide empiric antibiotic choices. Crude agreements between previous ETA with enrollment ETAs and with enrollment BALs were initially calculated separately. For the primary outcome, we also performed a sensitivity analysis where a positive BAL culture was defined as more than $10^{4}$ colony forming units/ 
$\mathrm{mL}$. Secondary outcomes included agreement matched to Gram class, organism and sensitivity profile, and potential antibiotic error rate (defined as $100 *$ [ 1 - crude agreement $]$ ).

\subsection{Statistical analysis}

Descriptive baseline statistics were performed on the entire sample, followed by a comparison between patients with and without prior cultures. Continuous variables were expressed as means and standard deviations and compared using $t$ tests. Categorical variables were compared using $\chi^{2}$ tests. Crude percent agreement with 95\% confidence intervals (CIs) was calculated for all patients who had positive prior and enrollment cultures, and $\chi^{2}$ tests were used to analyze subgroups stratified by antibiotic use, Gram class of prior cultures, and diagnostic technique. For patients with more than 1 previous culture drawn 1 to 3 days before enrollment, we pooled all specimens and considered all pathogenic organisms when calculating agreement. The frequency of individual organisms grown from previous and enrollment cultures was compared using a $\chi^{2}$ test. All analyses were performed using SAS version 9.1 (SAS Institute, Cary, NC).

\section{Results}

A total of 740 patients were randomized into the study; 1 patient withdrew consent within 2 days of randomization and was excluded from all analyses. Of the remaining 739 patients, 281 (39\%) had cultures done 1 to 3 days before enrollment. Patients with and without prior cultures had similar demographic and clinical characteristics (Table 1). Although patients with prior cultures had lower day 1 Multiple Organ Dysfunction Score [17] $(5.2 \pm 2.7$ vs $5.8 \pm 3.1)$, this absolute difference was likely not clinically important.

Of the 281 patients, $130(46 \%)$ grew a pathogenic organism on previous cultures. The most common organism isolated in previous cultures was Staphylococcus aureus, followed by Haemophilus influenzae, and Klebsiella

Table 1 Characteristics of patients with and without surveillance cultures at the time of enrollment

\begin{tabular}{|c|c|c|c|c|}
\hline & $\begin{array}{l}\text { Surveillance culture } \\
\text { done }(n=281)\end{array}$ & $\begin{array}{l}\text { No surveillance culture } \\
\text { done }(\mathrm{n}=458)\end{array}$ & $\begin{array}{l}\text { Total } \\
(\mathrm{N}=739)\end{array}$ & $P$ value \\
\hline Mean age \pm SD & $59.6 \pm 17.9$ & $58.6 \pm 17.7$ & $59.0 \pm 17.8$ & .35 \\
\hline \multicolumn{5}{|l|}{ Sex } \\
\hline Female & $83(29.5 \%)$ & $144(31.4 \%)$ & $227(30.7 \%)$ & .62 \\
\hline APACHE II $^{\mathrm{a}}$ & $19.9 \pm 6.2$ & $20.0 \pm 6.4$ & $20.0 \pm 6.3$ & .70 \\
\hline Days in intensive care & $7.6 \pm 4.0$ & $8.0 \pm 5.8$ & $7.9 \pm 5.2$ & .62 \\
\hline Days on mechanical ventilation & $7.4 \pm 4.1$ & $7.9 \pm 5.9$ & $7.7 \pm 5.3$ & .97 \\
\hline $\mathrm{PaO}_{2} / \mathrm{FiO}_{2}$ & $219.8 \pm 80.3$ & $215.5 \pm 84.1$ & $217.1 \pm 82.7$ & .37 \\
\hline \multicolumn{5}{|l|}{ Admission category } \\
\hline Medical & $170(60.5 \%)$ & $280(61.1 \%)$ & $450(60.9 \%)$ & \multirow[t]{2}{*}{.89} \\
\hline Surgical & $111(39.5 \%)$ & $178(38.9 \%)$ & $289(39.1 \%)$ & \\
\hline \multicolumn{5}{|l|}{ Primary diagnosis } \\
\hline Cardiovascular & $75(26.7 \%)$ & $106(23.1 \%)$ & $181(24.5 \%)$ & \multirow[t]{8}{*}{.58} \\
\hline Respiratory & $46(16.4 \%)$ & $82(17.9 \%)$ & $128(17.3 \%)$ & \\
\hline Gastrointestinal & $17(6.0 \%)$ & $43(9.4 \%)$ & $60(8.1 \%)$ & \\
\hline Neurologic & $38(13.5 \%)$ & $60(13.1 \%)$ & $98(13.3 \%)$ & \\
\hline Renal & $2(0.7 \%)$ & $6(1.3 \%)$ & $8(1.1 \%)$ & \\
\hline Sepsis & $9(3.2 \%)$ & $20(4.4 \%)$ & $29(3.9 \%)$ & \\
\hline Trauma & $77(27.4 \%)$ & $110(24.0 \%)$ & $187(25.3 \%)$ & \\
\hline Other condition & $17(6.0 \%)$ & $31(6.8 \%)$ & $48(6.5 \%)$ & \\
\hline MODS day $1^{\mathrm{b}}$ & $5.2 \pm 2.7$ & $5.8 \pm 3.1$ & $5.6 \pm 3.0$ & .02 \\
\hline \multicolumn{5}{|l|}{ No. of comorbidities } \\
\hline 0 & $84(29.9 \%)$ & $135(29.5 \%)$ & $219(29.6 \%)$ & \multirow[t]{4}{*}{.91} \\
\hline 1 & $70(24.9 \%)$ & $116(25.3 \%)$ & $186(25.2 \%)$ & \\
\hline 2 & $52(18.5 \%)$ & $93(20.3 \%)$ & $145(19.6 \%)$ & \\
\hline 3 & $75(26.7 \%)$ & $114(24.9 \%)$ & $189(25.6 \%)$ & \\
\hline \multicolumn{5}{|l|}{ Vasopressors } \\
\hline No & $228(81.1 \%)$ & $347(75.8 \%)$ & $575(77.8 \%)$ & \multirow[t]{2}{*}{.10} \\
\hline Yes & $53(18.9 \%)$ & $111(24.2 \%)$ & $164(22.2 \%)$ & \\
\hline \multicolumn{5}{|l|}{ Chest radiograph } \\
\hline New infiltrate & $76(27.0 \%)$ & $139(30.3 \%)$ & $215(29.1 \%)$ & \multirow[t]{2}{*}{.36} \\
\hline Worsening or persistent infiltrate & $205(73.0 \%)$ & $319(69.7 \%)$ & $524(70.9 \%)$ & \\
\hline
\end{tabular}

${ }^{\text {a }}$ APACHE II indicates Acute Physiology and Chronic Health Evaluation II [18].

b MODS indicates Multiple Organ Dysfunction Score [17]. 
pneumoniae (Table 2). The prevalence of individual organisms was similar in prior and enrollment cultures (Table 2). Of the 281 patients with prior cultures, 120 (43\%) patients had an enrollment culture with growth of pathogenic organisms not identified on prior cultures.

Among the 130 patients with positive prior cultures, 111 also had positive enrollment cultures. Agreement was similar if the enrollment culture was done via an ETA $(0.68$ [95\% CI $0.55-0.79])$ or BAL $(0.59[0.48-0.70], P=.33)$. Consequently, data from these 2 groups were combined for all subsequent analyses. There was no difference in agreement when a positive enrollment culture was defined using a quantitative threshold (only available for the BAL cultures) of more than $10^{4}$ colony forming units $/ \mathrm{mL}(0.70$ [0.59-0.79], $P=.16)$ compared to "any growth" definition of positive BAL culture. Agreement also remained similar between ETA (0.68 [0.55-0.79]) and BAL (0.70 [0.59-0.79], $P=.77)$ if a quantitative threshold was used to define a positive BAL culture.

Combining the results (regardless of diagnostic test, and using the "any growth" definition), in patients with positive prior cultures, we found overall agreement with all organisms in enrollment cultures to be $0.63(0.55-0.71)$. When only considering Gram stain class, agreement was 0.84 (0.77-0.89), and when sensitivity patterns were included, the agreement was $0.61(0.52-0.69)$ (Fig. 1). The potential antibiotic error rate was $16 \%(11 \%-33 \%)$ if agreement were based on Gram class, $37 \%(29 \%-45 \%)$ if based on presence of all organisms, and 39\% (31\%-48\%) when estimated according to sensitivity profile.

Patients prescribed new antibiotics during the surveillance period had better agreement (0.78 [0.64-0.87]) than patients on no antibiotics $(0.58$ [0.45-0.69]) or no new antibiotics (0.50 [0.32-0.68], $P=.033$ ) (Fig. 1). There was no significant difference in agreement between prior cultures

Table 2 Most frequent organisms grown on culture in patients with positive surveillance cultures

\begin{tabular}{lcc}
\hline Organism class & $\begin{array}{l}\text { Surveillance } \\
\text { culture results } \\
(\mathrm{n}=130)^{\mathrm{a}}\end{array}$ & $\begin{array}{l}\text { Enrollment } \\
\text { culture results } \\
(\mathrm{n}=130)^{\mathrm{a}}\end{array}$ \\
\hline Staphylococcus aureus & $40(30.8 \%)$ & $35(26.9 \%)$ \\
Hemophilus influenzae & $31(23.8 \%)$ & $28(21.5 \%)$ \\
Klebsiella spp & $17(13.1 \%)$ & $23(17.7 \%)$ \\
Enterobacter spp & $15(11.5 \%)$ & $23(17.7 \%)$ \\
Serratia spp & $10(7.7 \%)$ & $10(7.7 \%)$ \\
Escherichia coli & $8(6.2 \%)$ & $8(6.2 \%)$ \\
Acinetobacter spp & $6(4.6 \%)$ & $2(1.5 \%)$ \\
Pseudomonas spp & $6(4.6 \%)$ & $6(4.6 \%)$ \\
Methicillin-resistant & $5(3.8 \%)$ & $5(3.8 \%)$ \\
$\quad$ Staphylococcus aureus & $31(24 \%)$ & $22(16.9 \%)$ \\
Other & $0(0 \%)$ & $19(14.6 \%)$ \\
No growth & \multicolumn{3}{c}{} \\
\hline \multicolumn{2}{c}{ a Columns do not add to 130 because patients may have more than 1} \\
organism in the enrolment and surveillance cultures.
\end{tabular}

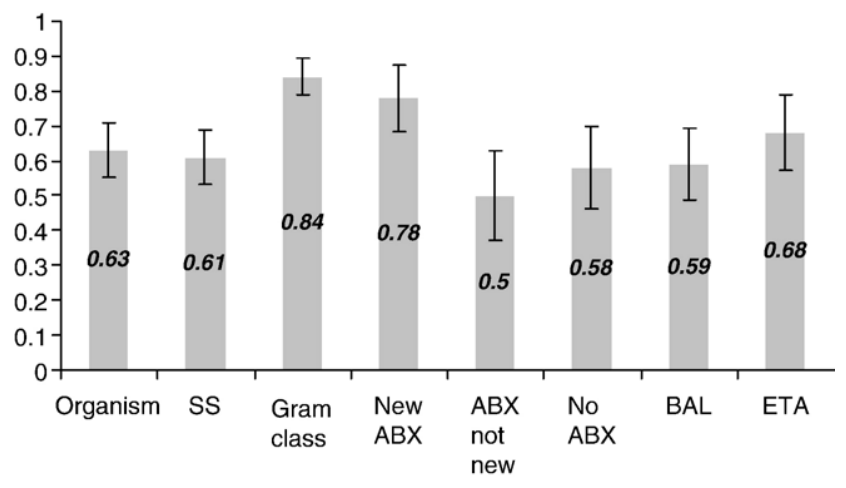

Fig. 1 Crude percent agreement and 95\% CIs between surveillance and enrollment cultures for different definitions of agreement and patient subgroups.

yielding Gram-positive organisms vs Gram-negative organisms (0.67 [0.49-0.82] vs 0.83 [0.72-0.90], $P=.11)$. Of the 4 most commonly isolated organisms, enrollment cultures were most frequently predicted by surveillance cultures for Serratia spp (0.9 [0.6-0.98]) and Staphylococcus aureus (0.82 [0.67-0.92]), and less frequently predicted with Klebsiella spp (0.57 [0.37-0.74]) and Enterobacter spp (0.61 [0.41-0.78]).

\section{Discussion}

This retrospective analysis of a large multicenter randomized trial suggests that previous respiratory cultures should not be used to guide empiric antibiotic choices for suspected ventilator associated pneumonia. We found that previous cultures failed to predict all organisms in enrollment cultures in $37 \%$ of patients, and a slightly higher failure rate occurs if one considers the proportion of patients growing the same bacteria with identical sensitivity profiles. If clinicians were to guide empiric VAP therapy based upon cultures drawn 1 to 3 days previously, they would potentially select inappropriate therapy $39 \%$ of the time, and even if they were to base empiric therapy on Gram class, they would still potentially select inappropriate therapy in $16 \%$ of cases. Given that outcomes of VAP have shown to be closely tied to adequacy of empiric antibiotics in retrospective observational studies [5, 6], these levels of agreement do not support the use of surveillance cultures to select empiric antimicrobials.

Surveillance and enrollment cultures may differ for various reasons. Prior antibiotic exposure may select out different causal organisms of VAP. However, agreement between surveillance and enrollment cultures was poor regardless of prior antibiotic exposure and was best in patients recently started on antibiotics. In patients who received no antibiotics 1 to 3 days before suspicion of VAP, it is possible that additional organisms grew in the following few days, leading to a high level of disagreement. Agreement may also depend on the same diagnostic method being used 
for surveillance and VAP diagnosis. We found slightly better agreement when ETAs were used for both specimens, but we were unable to analyze agreement when BALs were used to collect previous specimens because of very small numbers.

These results are consistent with other reports on surveillance cultures. Flanagan et al [11] reported that surveillance cultures predicted the organism grown at time of VAP diagnostic testing in only $11(65 \%)$ of 17 cases. Hayon et al [14] described 473 positive surveillance cultures in 125 episodes of microbiologically confirmed VAP. Only $16 \%$ of surveillance cultures predicted the causative organism [14]. However, Michel et al [10] have recently recommended systematic surveillance cultures based on a prospective cohort study using twice weekly ETAs. In their study, scheduled surveillance cultures predicted the correct organism in $34(85 \%)$ of 40 cases of VAP. They also assessed the utility of using these cultures to guide initial antibiotic coverage and found it to be adequate $96 \%$ of the time.

Several reasons could explain the discrepancy between our results and the high rates of agreement seen in the study by Michel et al. [10]. Their study was prospective, allowing clinicians to choose empiric antibiotics based on surveillance cultures. The clinician could use other patient features in addition to the surveillance cultures to make antibiotic choices and was free to broaden or narrow coverage, or to ignore the results of the surveillance culture. In our retrospective study, we could only calculate a potential rate of inadequate antibiotic treatment, and in clinical practice the rate of antibiotic adequacy would likely be higher due to clinical judgment, or by chance alone as even "narrow" spectrum antibiotics usually still cover multiple bacterial species. The study by Michel et al was also a small singlecenter study, which limits the generalizability of the results. Results of their study may be affected by the individual physicians prescribing the antibiotics or by the microbiology of their ICU. Finally, it is possible that the lower rate of agreement observed in our study was due to the low prevalence of resistant organisms such as Pseudomonas spp and MRSA in our patient sample. This low prevalence is partly attributable to the participating ICUs (predominantly Canadian) and because patients who were immunocompromised or colonized with Pseudomonas spp or MRSA were excluded from the study. Inclusion of patients already colonized with Pseudomonas spp or MRSA likely would have increased our rates of agreement.

Although our present study did not assess the adequacy of antibiotics directly, surveillance cultures failed to predict the correct Gram class in $16 \%$ of cases and the correct organism with identical sensitivity pattern in $39 \%$ of cases. This suggests that antibiotic selection based on surveillance cultures could be inadequate in up to $39 \%$ of cases. However, this finding should be interpreted cautiously because we did not directly study the consequence of doing, interpreting, and acting on surveillance cultures in clinical practice. In addition, surveillance cultures may still be useful by identifying patients colonized with resistant organisms and who may thus benefit from broader antibiotic coverage. Unfortunately, we were unable to assess this aspect of surveillance cultures due to the low prevalence of resistant organisms in our sample.

Because this study is a secondary analysis, it has several limitations. We have no information regarding the clinical reasons for ordering cultures before enrollment in the RCT. These may have been ordered for true surveillance in asymptomatic individuals, or patients may have had early clinical signs of VAP that precipitated the cultures. In the latter scenario, surveillance cultures may have represented early cultures from the same episode of VAP, which would tend to positively bias the agreement. Sampling bias could have also affected the results of the study. We analyzed only the 281 patients with surveillance cultures (of which only 130 had positive surveillance cultures) from a larger sample of 739 patients. It is possible that patients with surveillance cultures differed from those without such cultures, despite documented similarity in variables recorded (Table 1). However, unless regular surveillance cultures are routinely carried out, this is likely representative of typical clinical practice.

In conclusion, this secondary analysis of an RCT is the only multicenter study to assess agreement between prior cultures and cultures performed on the day of suspicion of VAP. We found poor agreement between prior cultures and the causative organism for VAP. Empiric antibiotics guided by prior cultures could lead to inadequate antibiotics in more than one third of cases. Prior culture results thus do not appear useful for guiding empiric antibiotic therapy. Further prospective studies using scheduled surveillance cultures are needed to confirm these results and to assess whether surveillance cultures in ICUs with high prevalence of resistant bacteria can identify patients where empiric therapy for VAP needs to include coverage of these bacteria.

\section{References}

[1] Chastre J, Fagon JY. Ventilator-associated pneumonia. Am J Respir Crit Care Med 2002;165(7):867-903.

[2] Heyland DK, Cook DJ, Griffith L, et al. The attributable morbidity and mortality of ventilator-associated pneumonia in the critically ill patient. The Canadian Critical Trials Group. Am J Respir Crit Care Med 1999;159(4 Pt 1):1249-56.

[3] Fagon JY, Chastre J, Vuagnat A, et al. Nosocomial pneumonia and mortality among patients in intensive care units. JAMA 1996;275 (11):866-9.

[4] Rello J, Ollendorf DA, Oster G, et al. Epidemiology and outcomes of ventilator-associated pneumonia in a large US database. Chest 2002;122(6):2115-21.

[5] Iregui M, Ward S, Sherman G, et al. Clinical importance of delays in the initiation of appropriate antibiotic treatment for ventilatorassociated pneumonia. Chest 2002;122(1):262-8.

[6] Kollef MH, Sherman G, Ward S, et al. Inadequate antimicrobial treatment of infections: a risk factor for hospital mortality among critically ill patients. Chest 1999;115(2):462-74. 
[7] American Thoracic Society, Infectious Diseases Society of America. Guidelines for the management of adults with hospital-acquired, ventilator-associated, and healthcare-associated pneumonia. Am J Respir Crit Care Med 2005;171(4):388-416.

[8] Rello J, Torres A, Ricart M, et al. Ventilator-associated pneumonia by Staphylococcus aureus. Comparison of methicillin-resistant and methicillin-sensitive episodes. Am J Respir Crit Care Med 1994;150 (6 Pt 1):1545-9.

[9] Raymond DP, Pelletier SJ, Crabtree TD, et al. Impact of antibioticresistant Gram-negative bacilli infections on outcome in hospitalized patients. Crit Care Med 2003;31(4):1035-41.

[10] Michel F, Franceschini B, Berger P, et al. Early antibiotic treatment for BAL-confirmed ventilator-associated pneumonia: a role for routine endotracheal aspirate cultures. Chest 2005;127(2):589-97.

[11] Flanagan PG, Findlay GP, Magee JT, et al. The diagnosis of ventilatorassociated pneumonia using non-bronchoscopic, non-directed lung lavages. Intensive Care Med 2000;26(1):20-30.

[12] Delclaux C, Roupie E, Blot F, et al. Lower respiratory tract colonization and infection during severe acute respiratory distress syndrome: incidence and diagnosis. Am J Respir Crit Care Med 1997;156(4 Pt 1):1092-8.

[13] Bouza E, Perez A, Munoz P, et al. Ventilator-associated pneumonia after heart surgery: a prospective analysis and the value of surveillance. Crit Care Med 2003;31(7):1964-70.

[14] Hayon J, Figliolini C, Combes A, et al. Role of serial routine microbiologic culture results in the initial management of ventilatorassociated pneumonia. Am J Respir Crit Care Med 2002;165(1):41-6.

[15] Canadian Critical Care Trials Group. A randomized trial of diagnostic techniques for ventilator-associated pneumonia. N Engl J Med 2006;355(25):2619-30.

[16] Heyland DK, Dodek P, Muscedere J, et al. Randomized trial of combination versus monotherapy for the empiric treatment of ventilator associated pneumonia. Crit Care Med 2008;36(2):1-8.

[17] Marshall JC, Cook DJ, Christou NV, et al. Multiple Organ Dysfunction Score: a reliable [18] descriptor of a complex clinical outcome. Crit Care Med 1995;23(10):1638-52.

[18] Knaus WA, Draper EA, Wagner DP, et al. APACHE II: a severity of disease classification system. Crit Care Med 1985;13:818-29. 